\section{Salaires des assistantes médicales pour 2002 et recommandations de salaires}

Etant donné que, depuis 1991, les sociétés cantonales de médecine élaborent leurs propres recommandations de salaires pour les assistantes médicales, nous renonçons désormais à publier des recommandations salariales pour l'ensemble de la Suisse.

Les conditions-cadres ci-après conservent toutefois leur valeur pour fixer individuellement le salaire d'une assistante médicale.

\section{Conditions-cadres}

\section{Formation}

- Diplôme de la Fédération des médecins suisses (estampille), resp. certificat de capacité de la FMH avec certificat de radioprotection et autorisation de faire des radiographies;

- Certificat fédéral de capacité d'assistante médicale.

Conditions de travail: bases de calcul

- Semaine de 43 heures en moyenne annuelle;

- 4 semaines de vacances (assistantes médicales de moins de 20 ans et de plus de 50 ans: 5 semaines).

Toute dérogation importante à ces conditions sera répercutée sur le salaire.

\section{Normes régionales}

Les salaires doivent être adaptés aux conditions régionales.

\section{$13^{\mathrm{e}}$ salaire}

Un $13^{\mathrm{e}}$ salaire doit être versé à l'assistante médicale à la fin de l'année. Si les rapports de service n'ont pas duré pendant toute l'année civile, le $13^{\mathrm{e}}$ salaire sera versé au prorata des mois d'engagement.

Prime d'ancienneté et augmentation du salaire réel Le montant de la prime d'ancienneté et d'une éventuelle augmentation du salaire réel est fixé au cours de l'entretien annuel sur les qualifications de l'employée.

Compensation du renchérissement

Les parties, conviennent à la fin de chaque année de l'octroi d'une compensation du renchérissement et, dans l'affirmative, de son envergure (ISPC: septembre: 0,7\%; octobre: 0,6\%).
Lorsque la compensation du renchérissement est convenue par contrat, elle doit être accordée.

Travail à temps partiel rétribué au mois Pour le travail à temps partiel, le salaire brut recommandé s'élève au 1/43 du salaire brut entier multiplié par le nombre des heures de travail hebdomadaire convenu (base de calcul: 43 heures hebdomadaires).

\section{Salaire horaire}

Un salaire horaire est recommandé en cas d'horaire de travail irrégulier et très réduit. Le taux horaire est de 6\%o du salaire mensuel. Il sera complété par une contribution aux vacances de 8,33\% correspondant aux quatre semaines de vacances habituelles (pour 5 semaines le taux sera de 10,64\% et pour 6 semaines $13,04 \%$, etc.).

\section{Heures supplémentaires}

En accord avec l'assistante médicale, l'employeur peut compenser les heures de travail supplémentaires dans les 14 semaines par des congés ou des vacances d'une durée au moins égale. Si elles ne sont pas compensées par des congés ou des vacances, l'employeur doit les rétribuer en versant le salaire normal, majoré d'au moins un quart. Le taux horaire est de 6\% d'un plein salaire mensuel, auxquels s'ajoute la contribution aux vacances (voir sous "Salaire horaire»).

\section{Les dispositions et recommandations suivantes s'appliquent à tous les salaires}

Déductions sur le salaire brut

- AVS, AI, APG, ACI: 6,55\% (AVS, AI, APG = 5,05\%, $\mathrm{ACI}=1,5 \%$;

- Assurance-accidents non professionnels: 1,367\% (pour un engagement de plus de 8 heures par semaine);

- Prévoyance professionnelle ( $2^{\mathrm{e}}$ pilier LPP): part de l'employée à la contribution (habituellement $50 \%$ ) calculée en fonction de l'âge, selon le certificat d'assurance.

Contrats de travail et autres conditions

d'engagement

Nous préconisons d'établir le contrat par écrit, sur la formule de la FMH créée conjointement avec les associations des assistantes médicales, accompagnée des recommandations cantonales. Ces documents peuvent être obtenus aux adresses suivantes:

- Fédération des médecins suisses FMH, Elfenstrasse 18, 3000 Berne 16, tél. 03135911 11;

- Association romande des assistantes médicales (ARAM), case postale 2034, 1002 Lausanne, tél. 02194345 64;

- Association suisse des assistantes médicales (SVA), case postale 6432, 3001 Berne, tél. 031 38114 43;

- Berufsverband Medizinischer Praxisassistentinnen (BMPA), Sonnenbergstrasse 7, 6005 Lucerne, tél. 04131022 23; 
- Fédération suisse des associations d'assistantes médicales (FSAAM), chemin du Lussy 18, 1806 St-Légier, tél. 0219434564.

L'Association d'assistantes médicales du canton de Genève a une convention collective de travail et l'Association du Tessin a son propre modèle de contrat de travail. Les deux sont élaborés avec les sociétés cantonales de médecine respectives et disponibles aux adresses suivantes:

- Association genevoise des assistantes médicales (AGAM), rue des Pavillons 11, 1205 Genève, tél. 02232110 69;

- Associazione Ticinese Assistenti di studio Medico (ATAM), Via Ronchetto, 6814 Cadempino, tél. 0919947835.

Les salaires des apprenties se basent sur les recommandations cantonales.
Délégués aux questions concernant les assistantes médicales

Dr Michel Marchev, président

Dr Jürg Kremo, vice-président

Pour la Fédération suisse des associations des assistantes médicales FSAAM (AGAM, ARAM, ATAM, BMPA)

Mme Elwina Kaufmann, présidente

Pour l'Association suisse des assistantes médicales SVA

Me Bruno Gutknecht, secrétaire central 